\title{
ON HERMITE-HADAMARD TYPE INEQUALITIES FOR HARMONICAL $h$-CONVEX INTERVAL-VALUED FUNCTIONS
}

\author{
Dafang Zhao, Tianding An, Guoju Ye and Delfim F. M. Torres
}

Abstract. We introduce and investigate the concept of harmonical $h$-convexity for intervalvalued functions. Under this new concept, we prove some new Hermite-Hadamard type inequalities for the interval Riemann integral.

Mathematics subject classification (2010): 26D15, 26E25, 28B20.

Keywords and phrases: Hermite-Hadamard inequalities, harmonical $h$-convexity, interval-valued functions.

\section{REFERENCES}

[1] M. U. Awan, M. A. Noor, M. V. Mihai, K. I. Noor, Inequalities associated with invariant harmonically h-convex functions, Appl. Math. Inf. Sci., 11 (2017), 1575-1583.

[2] Y. Chalco-Cano, A. Flores-Franulič, H. Román-Flores, Ostrowski type inequalities for interval-valued functions using generalized Hukuhara derivative, Comput. Appl. Math., 31 (2012), 457-472.

[3] Y. Chalco-Cano, W. A. Lodwick, W. Condori-Equice, Ostrowski type inequalities and applications in numerical integration for interval-valued functions, Soft Comput., 19 (2015), 3293-3300.

[4] Y. Chalco-Cano, G. N. Silva, A. Rufián-Lizana, On the Newton method for solving fuzzy optimization problems, Fuzzy Sets and Systems, 272 (2015), 60-69.

[5] T. M. Costa, H. Bouwmeester, W. A. Lodwick, C. Lavor, Calculating the possible conformations arising from uncertainty in the molecular distance geometry problem using constraint interval analysis, Inform. Sci., 415-416 (2017), 41-52.

[6] T. M. Costa, H. Román-Flores, Some integral inequalities for fuzzy-interval-valued functions, Inform. Sci., 420 (2017), 110-125.

[7] S. S. DRAGOMIR, Inequalities of Hermite-Hadamard type for functions of selfadjoint operators and matrices, J. Math. Inequal., 11 (2017), 241-259.

[8] A. Flores-Franulič, Y. Chalco-Cano, H. Román-Flores, An Ostrowski type inequality for interval-valued functions, IFSA World Congress and NAFIPS Annual Meeting IEEE, 35 (2013), 1459-1462.

[9] İ. İŞCAN, Hermite-Hadamard type inequalities for harmonically convex functions, Hacet. J. Math. Stat., 43 (2014), 935-942.

[10] İ. İşCAN, On generalization of different type inequalities for harmonically quasi-convex functions via fractional integrals, Appl. Math. Comput., 275 (2016), 287-298.

[11] M. KlaRiČIĆ BAKUla, K. NiKodeM, Converse Jensen inequality for strongly convex set-valued maps, J. Math. Inequal., 12 (2018), 545-550.

[12] M. A. Latif, S. S. Dragomir, E. Momoniat, Fejér type inequalities for harmonically-convex functions with applications, J. Appl. Anal. Comput., 7 (2017), 795-813.

[13] S. MARKov, Calculus for interval functions of a real variable, Computing, 22 (1979), 325-337.

[14] J. MatKows KI, K. Nikodem, An integral Jensen inequality for convex multifunctions, Results Math. 26 (1994), 348-353.

[15] M. V. Mihai, M. A. Noor, M. U. Awan, Trapezoidal like inequalities via harmonic h-convex functions on the co-ordinates in a rectangle from the plane, RACSAM, 111 (2017), 257-262. 
[16] M. V. Mihai, M. A. Noor, K. I. Noor, M. U. Awan, Some integral inequalities for harmonic h-convex functions involving hypergeometric functions, Appl. Math. Comput., 252 (2015), 257-262.

[17] F.-C. Mitroi, K. Nikodem, S. W A̧SOWICZ, Hermite-Hadamard inequalities for convex set-valued functions, Demonstratio Math. 46 (2013), 655-662.

[18] R. E. Moore, Interval analysis, Prentice-Hall, Inc., Englewood Cliffs, N.J., (1966).

[19] R. E. Moore, R. B. KeARfott, M. J. Cloud, Introduction to interval analysis, SIAM, Philadelphia, PA., (2009).

[20] K. Nikodem, J. SÁncheZ, L. SÁncheZ, Jensen and Hermite-Hadamard inequalities for strongly convex set-valued maps, Math. Aeterna 4 (2014) 979-987.

[21] M. A. Noor, K. I. Noor, M. U. AwAN, S. CostaChe, Some integral inequalities for harmonically h-convex functions, Politehn. Univ. Bucharest Sci. Bull. Ser. A Appl. Math. Phys., 77 (2015), 5-16.

[22] M. A. Noor, K. I. Noor, S. IfTIKHAR, C. Ionescu, Hermite-Hadamard inequalities for coordinated harmonic convex functions, Politehn. Univ. Bucharest Sci. Bull. Ser. A Appl. Math. Phys., 79 (2017), 25-34.

[23] M. A. Noor, K. I. Noor, M. V. Mihai, M. U. Awan, Fractional Hermite-Hadamard inequalities for some classes of differentiable preinvex functions, Politehn. Univ. Bucharest Sci. Bull. Ser. A Appl. Math. Phys., 78 (2016), 163-174.

[24] H. Román-Flores, Y. Chalco-Cano, W. A. Lodwick, Some integral inequalities for intervalvalued functions, Comput. Appl. Math., 37 (2018), 1306-1318.

[25] M. Z. Sarikaya, E. SET, M. E. ÖZdemir, On some new inequalities of Hadamard-type involving h-convex functions, Acta Math. Univ., Comenian LXXIX 2 (2010), 265-272. 